\section{Modified Newtonian Dynamics Close to Home}

IN HIS PERSPECTIVE "SEEING THROUGH DARK Matter" (3 August, p. 607), Stacy McGaugh describes the success of a modified Newtonian dynamics (MOND) in explaining the flat rotation curves of galaxies without invoking dark matter. In addition to noting that MOND is in accord with available data and observations, it is possible to directly test Newtonian dynamics in the laboratory, even at low accelerations. This has recently been done in a test of Newton's second law $(F=$ $m a$ ), and perfect agreement with Newton was found down to accelerations of $10^{-13} \mathrm{~m} / \mathrm{s}^{2}$, three orders of magnitude below the scale at which MOND should set in (1). Similarly, Newton's gravitational law has been tested to very small accelerations (i.e., with very small masses at small distances), and no deviations from the law were needed to describe the solar system (2). Thus, while no observational data disagree with MOND, recent laboratory tests indicate that Newtonian dynamics also explain galaxy rotation curves and apply to accelerations in the galaxy tails.

CHRISTOF M. AEGERTER

Fachbereich Physik, Universiät Konstanz, Universitätsstrasse 10, 78457 Konstanz, Germany.

\section{References}

1. J. H. Grundlach et al., Phys. Rev. Lett. 98, 150801 (2007).

2. J. Ciaverini et al., Phys. Rev. Lett. 90, 151101 (2003).

\section{Response}

CHRISTOF AEGERTER RIGHTLY POINTS OUT that no deviations from purely Newtonian behavior have been detected in the laboratory (1) to accelerations lower than the critical acceleration scale of MOND, where the mass discrepancy becomes evident in galactic systems. While a laboratory test of MOND would be highly desirable, Grundlach et al. (1) themselves point out that their result does not provide such a test. The reason is that their laboratory sits on the surface of the earth, where the acceleration we feel is 11 orders of magnitude above the MOND scale. It is the total acceleration that matters in MOND, so terrestrial experiments always exhibit Newtonian behavior even if their internal accelerations are arbitrarily small. 
A proper laboratory test of MOND requires that the apparatus itself be located in a region of very low acceleration, an extraordinarily difficult situation to arrange (2) on Earth. How far from Earth we need to be to detect MOND effects depends on the sharpness of the transition between the Newtonian and MOND regimes. In the optimistic case of a gradual transition, the Pioneer anomaly (the deviation of two Pioneer spacecraft in the outer solar system from their predicted trajectories) (3) might be a MOND effect $(4,5)$. Other solar system constraints (6 8) appear to favor a sharper transition, and the natural location for a clean experiment would be deep in intergalactic space. While that is obviously impossible, real laboratory tests are feasible. For example, in the relativistic extension of MOND hypothesized by Bekenstein [the tensor-vector-scalar gravity theory (9)], strong MOND effects are anticipated in regions where the gravitational potential nears zero. Such cancellation can be found at a point between the Sun and Earth (10), so a critical test is achievable with an appropriate satellite experiment.

\section{STACY MCGAUGH}

Department of Astronomy, University of Maryland, College Park, MD 20742 2421, USA.

\section{References}

1. J. H. Grundlach et al., Phys. Rev. Lett. 98, 150801 (2007)

2. A. Yu. Ignatiev, Phys. Rev. Lett. 98, 101101 (2007).

3. J. D. Anderson et al., Phys. Rev. Lett. 81, 2858 (1989).

4. M. Milgrom, New Astron. Rev. 46, 741 (2002).

5. M. E. McCulloch, Mon. Not. R. Astron. Soc. 376 (2007) 338.

6. R. H. Sanders, Mon. Not. R. Astron. Soc. 370, 1519 (2006).

7. M. Sereno, Ph. Jetzer, Mon. Not. R. Astron. Soc. 371,626 (2006).

8. J. Wallin, D. S. Dixon, G. L. Page, Astrophys. J. in press; preprint available at http://arXiv:0705.3408.

9. J. Bekenstein, Phys. Rev. D70, 083509 (2004).

10. J. Bekenstein, J. Magueijo, Phys. Rev. D73, 103513 (2006). 